\title{
TECHNOLOGY PRODUCERS USE OF LANGUAGE AND DISCOURSE TO SHAPE AND REINSTATE ANTI-BLACK GLOBAL REALITIES: \\ AN ANALYSIS OF AMAZON'S FACIAL RECOGNITION TECHNOLOGY COMMUNICATIONS AND RESPONSES TO RACIAL BIAS IN REKOGNITION
}

\author{
by \\ Charlotte Crawford, \\ Queen's University, Philosophy \& Gender Studies, 2015. \\ A MRP presented to Ryerson University \\ in partial fulfillment of the \\ requirements for the degree of \\ Master of Professional Communication \\ in the program of \\ Professional Communication \\ Toronto, Ontario, Canada, \\ (c) Charlotte Crawford, 2020
}




\section{Author's Declaration}

I hereby declare that I am the sole author of this MRP. This is a true copy of the MRP, including any required final revisions.

I authorize Ryerson University to lend this MRP to other institutions or individuals for the purpose of scholarly research.

I further authorize Ryerson University to reproduce this MRP by photocopying or by other means, in total or in part, at the request of other institutions or individuals for the purpose of scholarly research.

I understand that my MRP may be made electronically available to the public. 


\begin{abstract}
As of 2016, one in two American adults could be found in at least one American law enforcement face recognition network (Garvie, Bedoya \& Frankle, 2016). Racial bias in facial recognition technology is an important site of study as technology is largely conceived by public and state actors as neutral and democratic in nature, exempt from the biases and prejudices of human life (Noble, 2018). This study will trace the ways in which Amazon's responses to claims of racial bias in Rekognition and FRT general descriptions allow race and existing relations of power to manifest and persist. This study employs a critical discourse analysis to argue that Amazon works to obscure racial bias in both development and application of FRTs in law enforcement. Amazon also enables what I refer to as discourses of racial neutrality which, allows Amazon to deem any racially biased FRT outcomes as a "glitch in the system" that has nothing to do with race, despite decades of evidence proving otherwise.
\end{abstract}




\section{Dedications}

I would like to dedicate this major research paper along with all of my achievements and work to my wonderful father, Mark Crawford, who none of this is possible without. Thank you for unwavering support through all that I do, your hard work has allowed me to be here and write this. Also, to my supervisor Shana Almeida, whose teachings and work have enabled me to describe and analyze happenings of the world around me I knew to be true and felt for a long time. As anyone who has the privilege to learn from Shana knows, her contributions go beyond literature and the classroom as she empowers her students to identify and defend existing realities while never settling for less than we deserve. For that and so much more, I am forever grateful. This study is additionally only conceivable because of technology scholars like Safiya Umoja Noble, Joy Buolamwini, and Chris Wilson who join a long legacy of Black feminists and women who have dedicated their lives to showing the world that we will never be erased. 


\section{Table of Contents}

Author's Declaration ...........................................................................................................................i

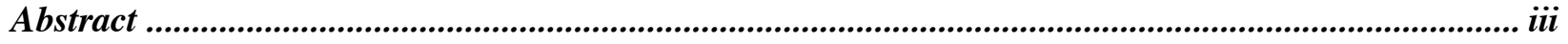

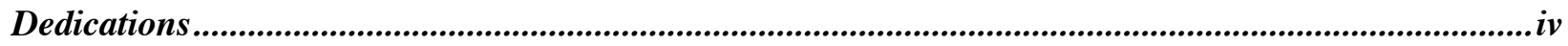

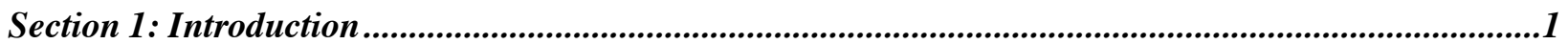

Amazon's Rekognition: A Case Study ………………………

Section 2: Literature Review ........................................................................................................................7

Lack of FRT Policy \& Standard Practice ..............................................................................................13

Racial bias in FRT: who and what develops it? ............................................................................14

FRT Detrimentally Targets Black Populations .................................................................................15

Gaps in Existing Literature .........................................................................................................19

Section 3: Theoretical Perspectives........................................................................................................22

Language as a Representation and Cultural Exchange........................................................................22

From Language to Discourse and Power .........................................................................................23

Technology and its Producers as Objective and Neutral...................................................................23

Critical Race Theory: Black Feminism as a Theoretical Approach to Technology..........................24

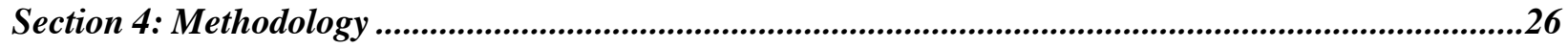

Data Collection: .....................................................................................................................................28

Section 5: Findings ......................................................................................................................29

Metaphors to Obscure Claims of Racial Bias in Rekognition.............................................................29

A Glitch in the System: Discourses of Racial Neutrality .......................................................................33

Relational and Expressive Value ……………………………...................................................33

Appeal to and Support of Dominant Ideologies around Law Enforcement and Black People.............35

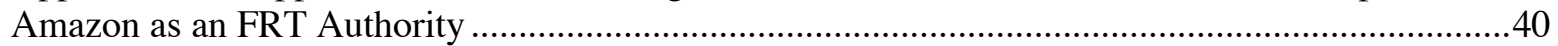

Discourses of Racial Neutrality .......................................................................................42

Amazon positioned as Ally to the Racial Other ................................................................43

Erasure of anti-Black Realities ..................................................................................................................45

Section 6: Conclusion ..................................................................................................................48

Section 7: Epilogue ................................................................................................................50

Section 8: Works Cited ..............................................................................................................52 


\section{Section 1: Introduction}

Facial recognition technology (FRT) has long been a part of our daily lives, from face ID on your iPhone to tagging your friend in a photo on Facebook. FRT can broadly be defined as a technological resource that can identify human faces based on their physiological or behavioural characteristics (DeMarco, 2012). The FBI has been using FRT in the US since 2011, yet there is still little publicly accessible information around how and where it is used (DeMarco, 2012). Discussions around the ethics of FRT have largely been absent from mainstream American public discourse until major tech corporations (Amazon, Google, IBM and Microsoft) sold their own FRTs to federal and state departments that proved to disproportionality misidentify people of colour (Samuel, 2018).

FRT research, particularly focused on its potential shortcomings, is crucial. As of 2016, one in two American adults could be found in at least one American law enforcement face recognition network (Garvie, Bedoya \& Frankle, 2016). The U.S. also has the highest prison rate per capita $(655$ per 100,000$)$ and prison population in the world $(2.12$ million) (Institute for Crime \& Justice Policy Research, 2019). These are important factors to know in relation to FRT because of how it used in a pipeline of AI technologies that can not only convict someone of a crime and put someone in jail, but also can determine their future employment, housing and travel opportunities (Buolamwini \& Gebru, 2018). Racial bias in FRT in particular is also an important site of study as technology is largely conceived by public and state actors as neutral and democratic in nature, exempt from the biases and prejudices of human life (Noble, 2018). This ideological framing of technology has been continuously circulated to silence the 
prevalence of racial bias in FRT. A critical, in-depth analysis of such claims and more importantly, their effects, is therefore much needed.

\section{Amazon's Rekognition: A Case Study}

Amazon is among the top five FRT producers in the world, next only to Microsoft, IBM ${ }^{1}$, Google, and Facebook (Horowitz, 2020). Amazon is primarily marketing and selling their FRT, Rekognition, to law enforcement agencies across the US, stating it is one of the most common uses for the technology (Cagle \& Ozer, 2018, para. 4). In what an Orlando police chief called a "first of its kind public-private partnership," Amazon provides free consulting, best practices coaching, and a mobile app for law enforcement agencies to build FRT "proof of concept cities" in the US (Cagle \& Ozer, 2018, paras. 4-7). Importantly, Rekognition has also proven to have the highest error rate in comparison to other dominant FRTs on those who are dark-skinned, particularly dark-skinned women (Kleinman, 2019). Amazon Web Services CEO Andy Jassy admitted the company has sold Rekognition to so many police departments and law enforcement agencies they do not know how many departments are currently using the technology or how it is being used (Gilbert, 2020, para. 3) Jassy also noted that Amazon’s Rekognition has “165 services in (the) technology infrastructure platform, and you can use them in any combination you want" (paras. 11). And while Google has withdrawn from FRT government sales and Microsoft has called for stronger FRT law enforcement safeguards acknowledging FRTs use and risks in racially biased practices and surveillance by law enforcement, Amazon has yet to follow

\footnotetext{
${ }^{1}$ IBM (June 2020) announced they are stopping all facial recognition technology research and development. This halt has and will not address the concerns of this study and is further discussed in Section 7: Epilogue.
} 
suit $^{2}$ (Doffman, 2019). Amazon and Rekognition therefore provides a compelling case study for critical analysis of how corporations respond to claims of racial bias in technology.

Importantly, critics of FRT have argued that the technology is being used to target already vulnerable Black communities (Buolamwini \& Gebru, 2018; Garvie, Bedoya \& Frankle, 2016, pp. 57). There are no statistical data to support that Black people are more likely to commit crimes or use illegal drugs in the US, yet Black Americans are five times more likely to be incarcerated, twelve times more likely to be convicted for drug possession and seven times more likely to be wrongfully convicted of a high-level crime than their White counterparts (Coles \& Powell, 2019). These high arrest rates cause Black people to be disproportionately represented in the mug-shot databases FRTs rely on (Garvie, Bedoya \& Frankle, 2016). Amazon states, "person tracking", national mugshot scanning, and "person of interest" investigations are made "easy and accurate" with Rekognition (Cagle \& Ozer, 2018, paras. 4-10). But Amazon's Rekognition communications fails to discuss how expediting these practices through FRT could impact already inordinate incarnation rates of Black people in the US.

In May 2018, the American Civil Liberties Union Foundation (ACLU) released “Amazon Teams Up With Government to Deploy Dangerous New Facial Recognition Technology," a study by Calge \& Ozer (2018) that not only highlighted racial bias in FRT development but also instances of Amazon's Rekognition being used to mass surveil citizens without consent based on Amazon's corporate interests. In June 2019, Amazon released a response in relation to ACLU's study (Calge \& Ozer, 2018) titled "Thoughts on Recent Research Paper and Associated Article on Amazon Rekognition". After the ACLU study was published,

\footnotetext{
${ }^{2}$ In June 2020, Amazon instated a one-year moratorium on Rekognition (Amazon's FRT) research and development within law enforcement after concerns of racial bias in FRT reached American mainstream media. This FRT hiatus by Amazon (further discussed in Section 7: Epilogue) does not address racial bias in Rekognition development and application nor does it mitigate the subsequent findings of this study.
} 
Amazon additionally updated the general description of Rekognition on their website, titled "The Facts on Facial Recognition with Artificial Intelligence" (2019).

This study will argue that Amazon's specific response to ACLU's study and their general description of FRT join a long history of racial discourses that work to maintain anti-Black existing relations of power and realities in America. Black people, throughout history, have dominantly and consistently been represented and Othered in American medias of authority, like news media or history books, as naturally violent or criminal (Hall, 1997). This representation or conception is constructed in direct contrast or opposition to the dominant portrayal of law enforcement agencies as innately good or saviours who naturally act in the best interest of a greater society. These hegemonic narratives around Black people and law enforcement actors then work to obscure claims of racial bias both in the application and development of FRT, as the racially biased and detrimental consequences the technology creates for Black communities are regarded as natural, normal or necessary (Hall, 1997). Further, law enforcement officers knowingly utilizing a technology that is proven to be racially biased in development or antiBlack in application is deemed unbelievable or an uncharacteristic event, even in the face of decades of evidence illustrating the opposite. This study will trace the ways in which Amazon's responses to ACLU's claims of racial bias in Rekognition and FRT general descriptions allow race and existing relations of power to manifest and persist, both materially (FRT production and development) and discursively (how technology use in law enforcement is communicated).

Amazon silences ACLU's and other claims of racial bias in the development and application of FRT in law enforcement while further supporting hegemonic ideologies that establish and maintain anti-Black realities for Black populations across the world. My research specifically seeks to answer the following questions: 1 . How does Amazon's description of 
Rekognition and their response to allegations of racial bias in Rekognition further obscure claims of racial bias in their FRT production and uses, and 2. How do Amazon's communications, both general and specific, contribute to the larger silencing of claims of anti-Black racism and violence inherent in the very reasons behind the use and development of FRT? 


\section{Section 2: Literature Review}

\section{FRT: Detection, Verification and Identification}

Facial Recognition Technologies or Face Recognition Technologies (FRTs) is an allencompassing term that generally refers to the use of "digital tools used to perform tasks on images or videos of human faces" (Buolamwini, Ordonez, Morgenstern \& Learned-Miller, 2020, pp. 2). FRTs can be further classified in three categories: face detection, face verification and face identification (Buolamwini et al., 2020). Recognizing the distinctions between and shortcomings built into these different types of FRTs is critical to understand the ways in which FRT is racially biased.

Buolammwini (et al., 2020) describe how face detection (FD) works to identify a face within a given picture or video; a necessary step for all subsequent FRT action, including face verification and face identification. False positives can occur in FD when an object is misidentified as a face or a face is missed (Figure 1). FD further allows for "face attribute classification and face attribute estimation," where the detected subject's race, gender, age and even perceived emotion and class are categorized based on their apparent facial expressions and distinctions (Buolamwini et al., 2020,pp.4). FD has proven to have practical challenges for Black populations, racialized communities and gender minorities specifically, since race and gender, as we understand them, are social constructs (Learned-Miller, Ordonez, Morgenstern \& Buolamwini, 2020). Consequently, facial classifications used in FD heavily rely on social, historical and cultural factors and conceptions that are not universally agreed upon or understood (Learned-Miller et al., 2020). Furthermore, FD false positives occur when a detected subject's attributes and/or facial expressions are misidentified or misunderstood (Figure 2) (Buolamwini et al., 2020). 
What is particularly important to note here about FD classifications is they are set or defined by FRT producers and developers (Buolamwini et al., 2020). For example, the characteristics or hallmarks that allow a detected face to be placed in the Black, Latinx, angry, "attractive" (Figure 2) or happy classifications within Rekognition are determined by Amazon's Rekognition developers and producers (Buolamwini et al., 2020). This is troublesome if we consider FRT production companies, along with the technology sector as a whole, is dominated by white men, who due to their lived experience and subjective knowledge do not fully understand or recognize the nuances, peculiarities and characteristics of Black, dark-skinned, racialized and gender minority faces and emotions (Noble, 2018). Such FRT classifications can be further utilized to "unfairly exclude or stereotype individuals who exhibit cultural characteristics deemed unfavorable by the (FRT) creators" (Buolamwini \& Gebrum, 2018, pp. 24). FD technologies from Microsoft, Amazon and IBM alike have accordingly proven to show lower performance rates on those who are darker-skinned, Black populations and women (Buolamwini et al., 2020). FD has further shown to be thirty-four percent less accurate on Black women who are dark-skinned in comparison to their white male counterparts (Buolamwini, 2018). This is evident in Figure 3, which shows that the face of MIT (Massachusetts Institute of Technology) and Algorithmic Justice League (AJT) FRT researcher, Joy Buolamwini (2016) who is a dark-skinned Black woman, is recognized by FD only when she wears a white mask. 

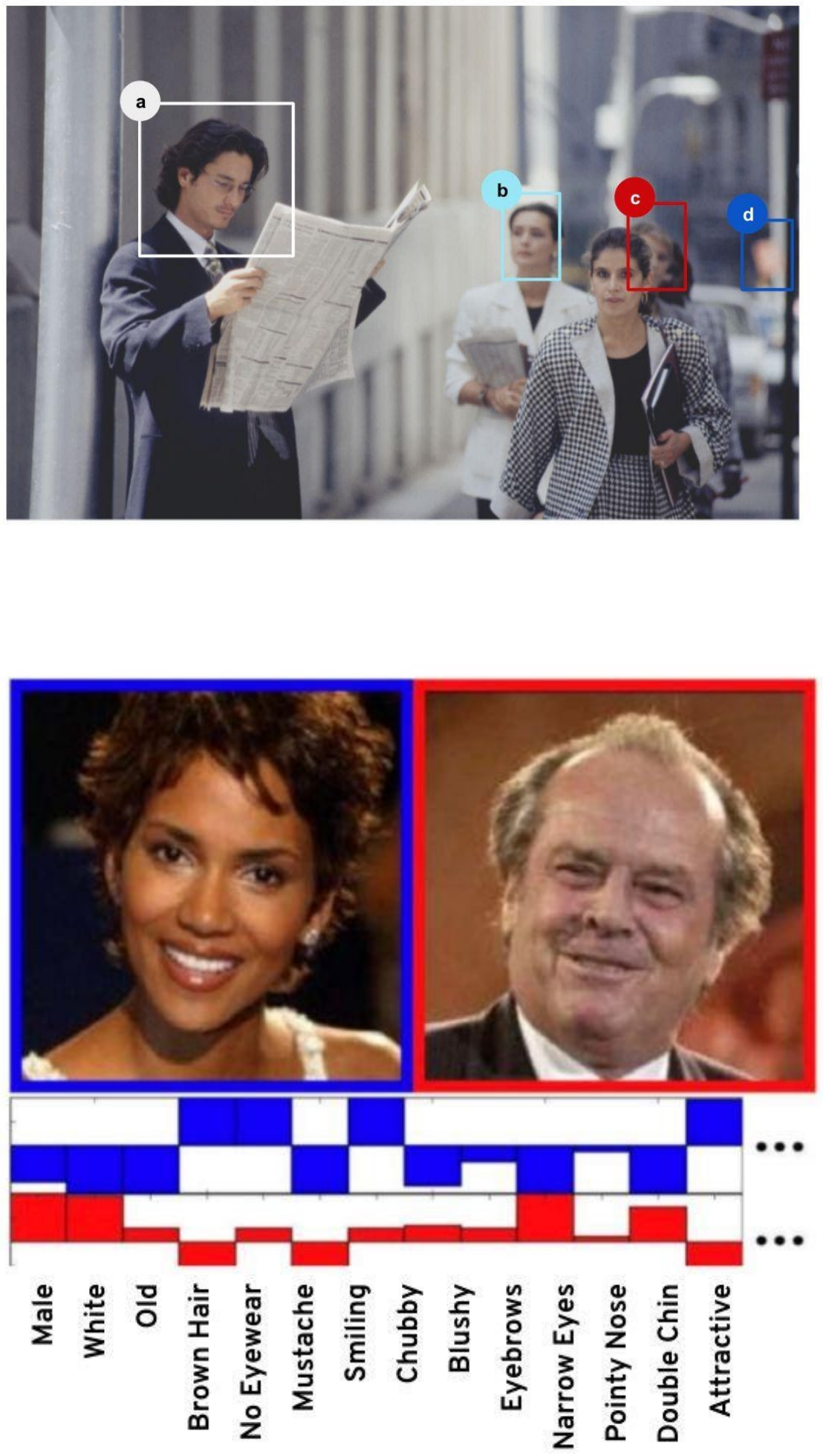

Figure 1: This figure illustrates how FD can miss faces present within an image (box $\mathrm{C}$ ) and misidentify objects as faces (box D). Retrieved from Buolamwini, J., Ordóñez, V., Jamie Morgenstern, \& Learned-Mille, E. (2020). Facial Recognition Technologies: A Primer. Algorithmic Justice League.

Figure 2: This figure demonstrates how FDs estimate facial attributes based on distinctions that are created and defined by FRT producers and developers, including Amazon. Retrieved from Kumar et al. as cited in Buolamwini, J., Ordóñez, V., Jamie Morgenstern, \& Learned-Mille, E. (2020). Facial Recognition Technologies: A Primer. Algorithmic Justice League. 


\section{Figure 3:}

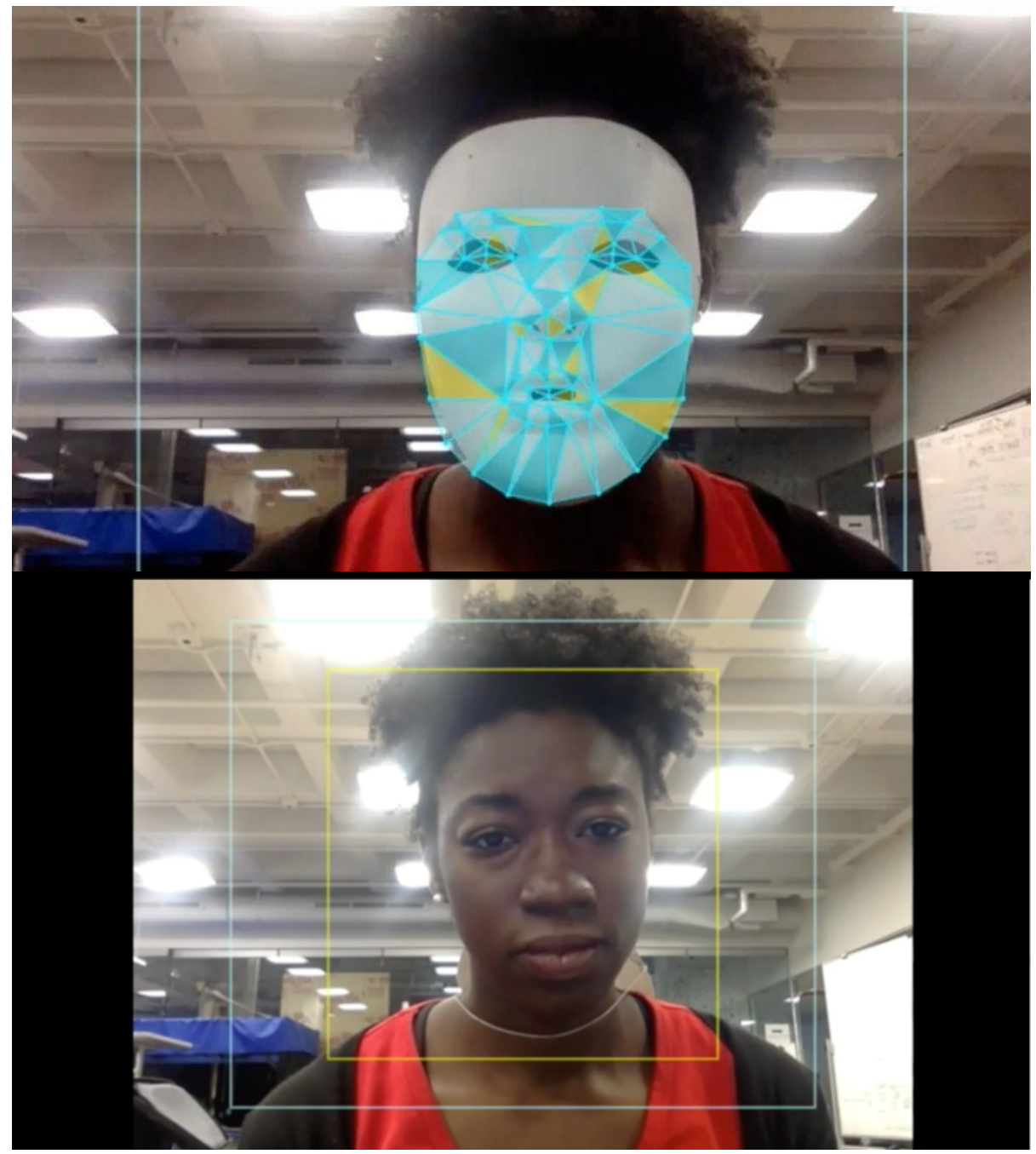

This figure shows Joy Buolamwini, a dark-skinned black woman, who is an FRT researcher at MIT and the Algorithmic Justice League. Her face is detected by the FD technology only when she wears a white mask. Retrieved from Buolamwini, J. (2016, November). How I'm fighting bias in algorithms. TedTalk, TEDxBeaconStreet.

Face verification (FV) moreover seeks to determine if a specific subject is present in a photo or video by a "1-to-1 comparison" (Buolamwini et al., 2020, pp. 3). FV is most commonly used for "access control" (Buolamwini et al., 2020, pp. 6). For instance, iPhone software takes a new photo of a user every time they use their face to open their phone and then utilizes FV to 
compare the photo to previously obtained information about the device owner's face to determine whether it is a match (Buolamwini et al., 2020). Face verification and detection alike are not entirely accurate or reliable as both can produce false matches due to image or video quality factors such as lighting, blur and angles (Learned-Miller et al., 2020).

Figure 4: This figure shows the kind of comparison that FV performs in order to determine if a given subject is in a photo. By comparing the facial attributes within the two photos, FV seeks to answer if the person on the left is the same as the person on the right (Buolamwini et al., 2020). Retrieved from Buolamwini, J., Ordóñez, V., Jamie Morgenstern, \& Learned-Mille, E. (2020). Facial Recognition Technologies: A Primer. Algorithmic Justice League.
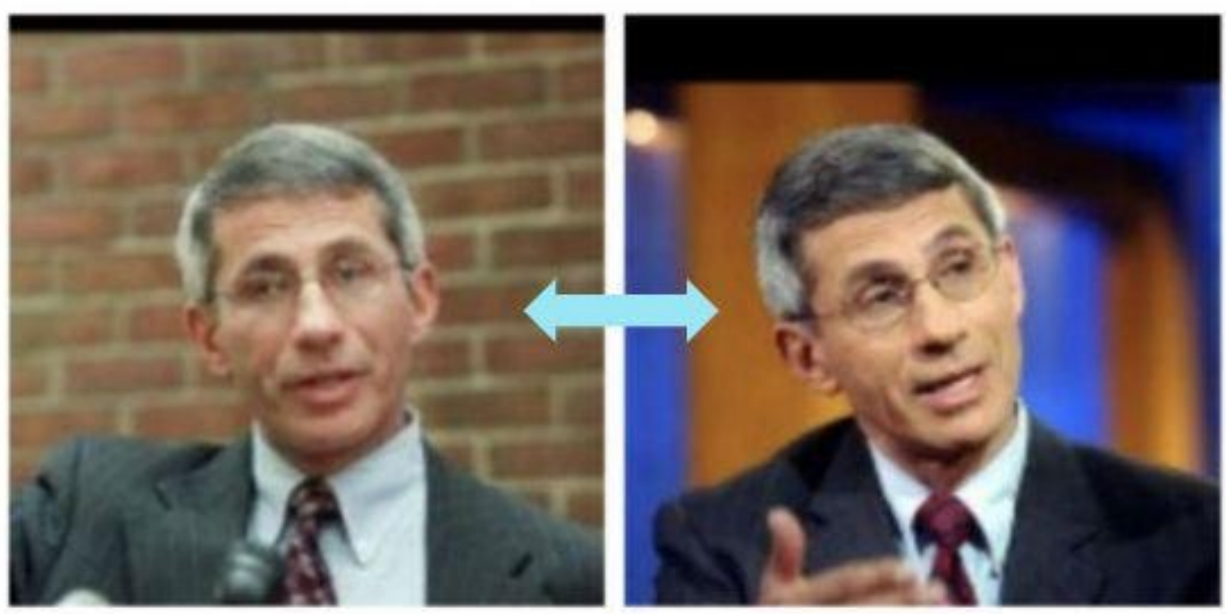

Lastly, facial identification (FI) works to discover who is in a photo based on comparing it to a gallery of images or a "1-to-many comparison" (Buolamwini et al., 2020, pp.9). Notably, FI can only "match the image of a face to a person for whom it already has some appearance information" (Buolamwini et al., 2020, pp. 6). Therefore, entirely accurate results in FI can only occur if the subject's image entirely matches one within its existing gallery (Learned-Miller et al., 2020). The success and effectiveness of FI systems are then entirely dependent on existing systems and their components (Buolamwini et al., 2020). 
As Noble (2018) writes, this is contrary to how FRTs are dominantly discussed in the public sphere, as a force that improves existing operational standards and mitigates historical practical shortcomings of law enforcement agencies. In reality, FRTs do not rectify existing systems including biased practices, rather they expedite and escalate them. This is further made problematic in application as FI systems in FRTs are used as justification for life-altering law enforcement practices such as "real-time video surveillance, arrest and identify, identify and arrest, investigate and identify, and targeted searches" (Garvie, Bedoya \& Frankle, 2016, pp. 1012).

There is evidently no type of FRT that is without vast possibility and likelihood of error, especially for Black communities (Learned-Miller et al., 2020). It is simply not possible for any FRT technology, as it currently exists, to independently produce a person's "unique faceprint" (Buolamwini et al., 2020, pp. 12). Joy Buolamwini (2016) therefore coined the term "the coded gaze," which speaks to the ways in which AI-powered technology, including all types of FRT, reflect "the priorities, the preferences, and also sometimes the prejudices of those who have the power to shape technology" (4:52). As no FRT technology is without deficiencies or inaccuracies, FRT producers are empowered to deem which shortcomings are most desirable or appropriate when selecting FRT systems based on their own coded gaze (Buolamwini et al., 2020). FRT producers do not fail to consistently mention the technology's innate shortcomings by accident - but rather this is a feature of what Gates (2011) refers to as Western neoliberal project that seeks to commodify every aspect of society, including identities, to maintain existing hegemonic relations of power with or without the knowledge of citizens. 


\section{Lack of FRT Policy \& Standard Practice}

Technological developments and advancements have historically always moved faster than legislation can keep up with, allowing tech corporations to employ Facebook CEO Mark Zuckerberg's famous motto to "move fast and break things" (Taneja, 2019, paras. 1-2). Without any FRT specific legislation, holding tech corporations accountable for FRT misuse is difficult, despite its widespread law enforcement and commercial use (DeMarco, 2012). One of the most documented concerns throughout FRT literature is that there is no comprehensive federal law around the collection and use of personal information and digital data at large (Buolamwini \& Gebrum, 2018; Garvie, Bedoya \& Frankle, 2016; Introna \& Nissenbaum, 2010; Jolly as cited in Brinckerhoff, 2012; Noble, 2018). Instead, there is a patchwork of state-specific data privacy laws and best practice guides that differ by industry (Brinckerhoff, 2012).

As previously mentioned, there is no single type of FRT has proven to be most reliable or without error; therefore, this lack of standardized FRT policy increasingly allows for a lack of consistent FRT operational standards and practice (Buolamwini et al., 2020; Introna \& Nissenbaum, 2010). Each FRT operator is then empowered to not only define and determine facial classifications, which encoded shortcomings are most desirable as discussed but also, their own benchmarks (Buolamwini et al., 2020, pp. 12; Introna \& Nissenbaum, 2010). FRT benchmarks can be understood as the decided threshold of similarity between the probe and gallery image, which is determined by the user (Introna \& Nissenbaum, 2010). Developing new FRT regulation is then challenging as every system is different, allowing law enforcement agencies to self-regulate their own FRT use. Furthermore, this positions FRT producers like Amazon as the sole evaluators of their own technology and its subsequent implications (Introna \& Nissenbaum, 2010). 


\section{Racial bias in FRT: who and what develops it?}

There are various theories about the origin of racial bias in FRT. Some technology scholars argue it starts with the developer intentionally or unintentionally using biased coding practices in the creation of the base algorithm (Barocas \& Selbst, 2016; Noble, 2018; LearnedMiller et al., 2020; Buolamwini et al., 2020). As mentioned, technology and software development are White-cisgender-male dominated industries, due to sexist, racist and transphobic social relations and structures of power (Noble, 2018). Encoded FRT racial bias is then inevitable if we consider who produces the software (Noble, 2018). Racial bias within FRT is propagated in application as the technology relies on problematic data practices within technology corporations that historically centre the experiences and needs of White men (Learned-Miller et al., 2020).

Many scholars argue that it is in fact the training data, the data the starting algorithm engages with, that produces a racial bias in FRT (see for example Caliskan, Bryson, \& Narayanan, 2017; Introna \& Nissenbaum, 2010; Silva \& Kenney, 2018). FRT base algorithms made seemingly neutral by automated parsing of web data sets alone can pick up bias from the data itself (Caliskan, Bryson, \& Narayanan 2017). Noble (2018) argues that once an FRT's algorithm learns a prejudice as correct from its database, it will continue to infer data rules, patterns and predictions that hold that same prejudice, especially when that prejudice is further solidified by new incoming data. Silva \& Kenney (2018) also assert it is not the physiological makeup of an individual's face that identifies them in FRT but rather an algorithm's statistical training data learning. Training data then becomes the basis of validity for FRT, so subsequent data that aligns with that initial data is further reproduced as it is deemed correct (Noble, 2018). Racial bias in FRT begins in the starting algorithm and is then further exasperated in its growth and development, the training data (Buolamwini et al., 2020; Learned-Miller et al., 2020; Noble, 
2018). This is important for this study as many technology producers, like Amazon, claim that racial bias and potential for error in FRT are both reduced over time, as the algorithm interacts with consumer data. But it is in fact the opposite (Buolamwini et al., 2020; Caliskan, Bryson, \& Narayanan, 2017; Introna \& Nissenbaum, 2010; Learned-Miller et al., 2020; Noble, 2018; Silva \& Kenney, 2018).

Racial bias within FRT is evidently multi-faceted in its manifestation. Accordingly, national or widespread benchmarks or operational standards will not entirely fix the issue (Learned-Miller et al., 2020). Operational standards are not enough as the reliability and success of FRT systems are entirely dependent on deployment factors (Buolamwini et al., 2020). Therefore, FRT legislative operational standards or benchmarks will only accurately indicate or regulate FRT operations in the exact conditions represented in the benchmark data (LearnedMiller et al., 2020). As there is an unlimited set of conditions where FRT can be used, even within law enforcement, legislative benchmarks could only, at best, account for a small set or limited amount of practical conditions (Learned-Miller et al., 2020).

\section{FRT Detrimentally Targets Black Populations}

Black people are more likely to be stopped, interrogated, and investigated by police and subjected to facial recognition software than any other demographic in the U.S. (Buolamwini \& Gebru, 2018; Garvie, Bedoya \& Frankle, 2016). In Minnesota, for example, Black people represent $5.4 \%$ of the population; however, they represent $24.5 \%$ of the state's arrests (Garvie, Bedoya \& Frankle, 2016). In comparison, their White counterparts in Minnesota represent $82.1 \%$ of the population, but $57 \%$ of arrests (Garvie, Bedoya \& Frankle, 2016). As mentioned, Black people then disproportionately dominate the existing law enforcement image galleries and databases that FRTs rely on (Garvie, Bedoya \& Frankle, 2016). Of concern for Black 
populations, FRTs have consistently proven to be better at catching facial differences in those who are White or lighter-skinned and consistently have the lowest accuracy rate for those who are Black or dark-skinned (Buolamwini \& Gebrum, 2018; Garvie, Bedoya \& Frankle, 2016; Introna \& Nissenbaum, 2010; Learned-Miller et al., 2020). IBM's facial recognition technology error rate is seven times higher on Black people and in particular 20\% higher on Black women (Buolamwini \& Gebrum, 2018). In addition, in subsequent test by the ACLU Amazon's Rekognition misidentified twenty-eight members of Congress as people who have been arrested for a crime (Snow, 2018). Black members make up only $20 \%$ of Congress yet accounted for $40 \%$ of Rekognition's software misidentification errors (Snow, 2018). FRT's disproportionate error rate on those who are dark-skinned has proven to have injurious real-world implications for Black Americans like Robert Williams - who was arrested at his home in front of his wife and daughter without reason after Detroit police department's FRT misidentified him as a shoplifter (Garcia-Hodges, Sottile \& Ward, 2020). Williams was held in custody without cause for over thirty hours, "humiliated...fingerprinted and mugshotted before anybody asked (him) one question," and was only released (but not removed from Detroit's law enforcement system) once the arresting police officers acknowledged "the computer had it wrong" (Garcia-Hodges, Sottile \& Ward, 2020, paras. 2-8).

FRTs have further proven to be more likely to overestimate performance on homogenous galleries, as it becomes harder to determine distinct facial differences (Learned-Miller et al., 2020). The harms of FRT misidentification thus tend to fall heavily on low-income Black communities in particular (Buolamwini et al., 2020; Buolamwini \& Gebrum, 2018; Garvie, Bedoya \& Frankle, 2016; Introna \& Nissenbaum, 2010; Learned-Miller et al., 2020; Noble; 2018). FRTs have also been used to "identify the criminality of a person from their facial image" 
based on existing databases of criminal facial attributes (Harrisburg University, 2020, para. 7). If we consider who is consistently produced or regarded as criminal, this use of FRT directly works to target and further incarcerate and criminalize Black members of society (Learned-Miller et al., 2020). This is made further possible by both existing and historic anti-Black law enforcement practices and the lack of national FRT policy and regulation (Garvie, Bedoya \& Frankle, 2016). Like other algorithm-powered technologies, FRT learns from our practices, behaviours and policies; it mimics our patterns and biases (Buolamwini \& Gebrum, 2018). Contrary to dominant ideologies, technology is not made in a vacuum. If you "put garbage (anti-black racism) in you'll get garbage (anti-black racism) out" (Barocas \& Selbst, 2016). This is compounded when we consider that FRT, like all technology, is publicly conceived of as neutral and as such is often given more authority in practice than human decision making (Learned-Miller et al., 2020;

Noble, 2018).

\section{Figure 5:}

\section{Ratio of African American arrest rates to population share in select jurisdictions.}

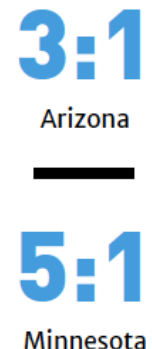

Minnesota

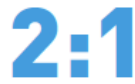

Hawaii

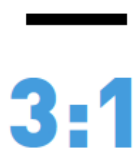

Pennsylvania

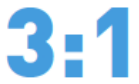

L.A. County

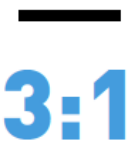

San Diego County

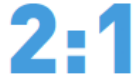

Michigan

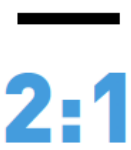

Virginia

Sources: U.S. Census, Minnesota Department of Public Safety, King County Department of Adult and Juvenile Detention, Pennsylvania Uniform Crime Reporting System, State of California Department of Justice Office of the Attorney General, Virginia State Police, Arizona Department of Public Safety ${ }^{236}$

This figure illustrates the disproportionate arrest rate of Black people across states in America, showing why they are overrepresented in the FRT galleries and databases used by law enforcement agencies. Retrieved from Garvie, C., Bedoya, A., \& Frankle, J. (2016). The 
Perpetual Line-Up: Unregulated Police Face Recognition in America. Georgetown Law Center on Privacy \& Technology.

\section{FRT-powered Mass Surveillance without Consent}

FRT is also a biometric technology—it utilizes automated systems to determine a person's unique features (Buolamwini et al., 2020). What distinguishes FRT however from other biometric technologies such as fingerprint scanners, is its ability to be used from a distance, without disclosure or consent of citizens (Buolamwini et al., 2020). Law enforcement agencies can then utilize FRT to ignite mass surveillance on given populations or subjects without their knowledge (Buolamwini \& Gebrum, 2018). This is an advantageous practice for law enforcement agencies, as the widespread effectiveness of FRT is reliant on the building a gallery of images for face identification (Learned-Miller et al., 2020).

Furthermore, FRT is employed for demographic targeting, which seeks to "create demographic and biometric profiles of individuals based on facial features" (Learned-Miller et al., 2020, p. 6). FRT facilitates demographic targeting through autonomous video and photo surveillance in public spaces (Learned-Miller et al., 2020). This practice has only increased in the U.S. since the passing of the Patriot Act in 2011, post 9-11 (Joseph \& Lipp, 2018). The Patriot Act still today allows government and law enforcement agencies to surpass legal regulations and infringe on the human rights of citizens under the veil of protecting national security (Rubel, 2007). For example, the NYPD started an FRT program under the Patriot Act to video monitor and take pictures of all those who enter New York's subway systems without the knowledge or consent of the public in order "to keep this city safe" (Joseph \& Lipp, 2018, para. 8). This allows local police and law enforcement agencies to quickly search through mass amounts of video footage to locate suspects based on internal classifications of facial features, emotions, hair type and skin tone (Joseph \& Lipp, 2018). "Whenever there has been the creation 
and enforcement of categories, there has been surveillance" mediated by societally dominant beliefs, conceptions and practices (Graham \& Wood, 2003, p. 227).

As Jerome Greco, a digital forensics staff attorney at the Legal Aid Society in New York stated:

"I imagine a scenario where a vague description, like young black male in a hoodie, is fed into the system, and the software's undisclosed algorithm identifies a person in a video walking a few blocks away from the scene of an incident... The police find an excuse to stop him, and, after the stop, an officer says the individual matches a description from the earlier incident. A man who was just walking in his own neighborhood could be charged with a serious crime, without him or his attorney ever knowing it all stemmed from a secret program which he cannot challenge" (as cited in Joseph \& Lipp, 2018).

Real-time video and photo surveillance in public spaces without public knowledge is not specific to New York or the NYPD. Almost all FRT producers, including Amazon, offer realtime video services (Garvie, Bedoya \& Frankle, 2016). One hundred and seventeen million Americans can be found within a law enforcement FRT gallery in the U.S. (Garvie, Bedoya \& Frankle, 2016). Police officers in Washington County reported using Amazon's Rekognition on average 20 times per day (Washington Post, 2019). Many FRT systems used by law enforcement produce "top matches," no matter how accurate they are, as benchmarks are internally set (Garvie, Bedoya \& Frankle, 2016, pp.54). FRT algorithms are additionally not tested for racial bias, despite the many studies that have proven they underperform on those who are darkskinned (Garvie, Bedoya \& Frankle, 2016). Therefore, "if the suspect is African American rather than Caucasian, the system is more likely to erroneously fail to identify the right person, potentially causing innocent people to be bumped up the list - and possibly even investigated" (Garvie, Bedoya \& Frankle, 2016, pp.54).

\section{Gaps in Existing Literature}

Existing FRT literature clearly identifies the ways in which racial bias becomes a part of FRT and discusses how FRT's application reproduces existing structures of power, further 
marginalizing vulnerable communities like Black Americans. Even though there is growing evidence of the ways in which wide FRT adoption by law enforcement agencies disproportionally produces detrimental consequences for Black communities this is still not publicly discussed by FRT or technology producers. What is missing from existing FRT literature is how major tech corporations' responses to allegations of racial bias in FRT works to maintain existing racist hegemonic relations of power. My research project will address this gap highlighting how Amazon's response to racial bias allegations in Rekognition continues to reproduce hegemonic relations of power by further silencing members of the Black communities who experience discrimination and violence at the hands of police and law enforcement agencies.

Furthermore, there is a lack of FRT research on the implications of major tech corporations' responses to allegations of racial bias in FRT within the Canadian context. Similar to the U.S., Canada has no national FRT policy, regulation, benchmarks or operational standards (CBC, 2020). The RCMP (Royal Canadian Mounted Police) along with the police departments of Vancouver, Edmonton, Calgary, Toronto and Halifax Regional, have been using FRT without public knowledge and only publicly confirmed the use of the technology after evidence was publicly reported by the CBC (2020). As American corporations supply Canadian law enforcement agencies with FRT, the same encoded racial bias is then duplicated here in Canada (CBC, 2020). Additionally, similar to the U.S., anti-Black relations and structures of power, especially in law enforcement, persist in Canada. In Toronto, Black people are over-represented in stop, arrest, and investigation rates and "in use of force cases (28.8\%), shootings (36\%), deadly encounters (61.5\%) and fatal shootings (70\%)" (Ontario Human Rights Commission, 2018, para 1). The TPS (Toronto Police Service) has also participated in carding and other forms 
of racialized surveillance and arrest practices (Allen \& Gillis, 2019). As interactions with the police and law enforcement are more likely to occur and be deadly for Black Canadians (Ontario Human Rights Commission, 2018), there must be further investigation into the role of tech corporations', American and Canadian alike, communications and responses to allegations of racial bias in their technology and how this works to maintain anti-Black relations of power in Canada.

My research project will address these gaps focusing on how Amazon's responses to racial bias allegations in Rekognition work to reproduce discursive, hegemonic relations and structures of power, in the U.S. and Canada, by further silencing members of the Black communities who experience discrimination and violence at the hands of police and law enforcement agencies who use FRT. 


\section{Section 3: Theoretical Perspectives}

\section{Language as a Representation and Cultural Exchange}

Stuart Hall (1997) centralizes the idea of culture in how we come to understand the world around us. He asserts those who come from a shared culture have a set of "shared meanings" or common-sense understandings that allow them to interpret the world in similar ways (p. 2). This is not to infer those from a shared culture necessarily perceive the world in the same way. Rather, culture organizes and regulates our devotions, emotions, conceptions and ideas through the ways in which objects and subjects are continuously represented in various visual, written and other media such as TV, news media, corporate responses, books or policy (Hall, 1997). Language, Hall argues, is a "system of representation" (p. 1) that assigns, symbolizes and disseminates meaning to both create and maintain shared culture. It is through "cultural codes" or shared concepts, ideas and images assigned to language, subjects and objects that enable people of a shared culture to interpret the world through roughly similar ways. Importantly, Hall also argues that culture is an interactive and ongoing practice that reinforces the production, reproduction and exchange of dominant and shared understandings in a given society.

Hall discusses the traffic light to exemplify this idea. Those from a shared culture have a similar understanding of a traffic light; not because of the traffic light's intrinsic properties, but because of the meaning the traffic light carries and reproduces, through personal and social codes. Hall's conception of language as a cultural exchange thus implies that representations are the primary site of meaning and knowledge production that allows us to negotiate how we move through the world around us. 


\section{From Language to Discourse and Power}

Language is an operational representational system whose investments are clear by examining the ways in which discourse is constructed (Hall, 1997). To understand this process, we must first define ideologies, which are societal common-sense understandings that are embedded with the interests, values and conventions of those in power (Fairclough, 2015). Ideologies shape the ways in which we understand the world around us and the people in it, they are given more power the more they circulate (Almeida, 2020). Discourse can then be understood as a social practice, a "ideological force," and the "production of knowledge through language" and shared cultural meanings (Simpson, Mayr, \& Statham, 2018, p. 29; Hall, 1992, p. 44). It is an assortment of circulated text and images that define and regulate social relations, topics, subjects and ideas, always in relation to power (Foucault, 1980).

\section{Technology and its Producers as Objective and Neutral}

Technology is predominately represented throughout public discourse as not only neutral or objective in nature but a democratizing force, giving voices to communities that have long been historically silenced (Introna \& Nissenbaum, 2010). Technology is conceived as neutral throughout dominant discourse as it is thought to be rooted in objective mathematics and science (Noble, 2018). What is not often represented in governing discussions of technology is the proven biases of those who produce it (Noble, 2018). The financial interests of technology corporations and the ways in which they directly influence and determine technological development and knowledge formation is largely absent from the public domain (Noble, 2018). What Safiya Noble (2018) coined as algorithmic oppression, highlights the structural ways in algorithmic-driven data and communications reinforces fundamental racism and oppressive hegemonic ideologies. 
This is similar to what Daston and Galison (1992) referred to as the perceived "mechanical objectivity" of scientific atlases or collections of data. Such images are riddled with idiosyncrasies even though they are defined or perceived as censoring the personal (Daston \& Galison, 1992). Those who produced scientific atlases or tables in the early twentieth century were enabled to decide which scientific images and accounts represented "what truly is," based on their own judgements or understandings of truth (Daston \& Galison, 1992, p. 84) This worked to produce certain objects and subjects in a manner that was deemed characteristic or typical in order to "make nature safe for science" Daston \& Galison, 1992, p. 85). This worked to replace raw experience within the data we refer to as the objective base for science today with digestible and uniform atlases and images (Daston \& Galison, 1992).

\section{Critical Race Theory: Black Feminism as a Theoretical Approach to Technology}

Critical race theory and Black feminism alike regard the social, political and historical as intrinsically tied and central to how we come to understand topics, subjects, objects and ideas (Noble, 2018). Black feminism as an approach to technology highlights the stereotypes and racial hierarchies that are engrained and consistently produced and reproduced in technology like FRT and the data it develops (Noble, 2018). This is critical as how and what information is within dominant discourse is directly influenced by existing social relations of power and is further central to human freedom and development (Noble, 2018). As Noble (2013) states, "technologies themselves and their design do not dictate racial ideologies; rather, they both reflect and re-instantiate the current social climate and prevailing social and cultural values" (para. 8). 
The aforementioned theories of representation, discourse, culturally dominant ideologies of technology as neutral, and critical race theory will be applied to this study of Amazon's general communications and specific responses around racial bias in FRT. Together, these theories provide a framework for understanding the ways in which mechanisms of power, whether discursive (Amazon's responses to and communications around racial bias in Rekognition) or material (Rekognition or FRT technology itself), work to reproduce dominant representations of race. 


\section{Section 4: Methodology}

Critical discourse analysis (CDA) is the chosen method for this study as it highlights the ways in which those in power manage their projects through language and public discourse (Fairclough, 2015). Fairclough (2015), founded CDA to illuminate the ways in which discourse is an ideological force that develops and maintains "common-sense assumptions" and shapes our beliefs, knowledge and behaviour (p.13). Ideologies and discourse are both closely linked to power, as they are "a means of legitimizing existing social relations and differences of power" (p.2) and contribute to existing unequal power relations in varying degrees (Fairclough, 2015). The ideological implications of discourse are then not usually not obvious or clear (Fairclough, 2015). As Fairclough (2015) states, "ideology is most effective when its workings are least visible" (p. 108). Those within a given culture regard, consciously or unconsciously, the hegemonic ideologies entrenched within discourse as common-sense or natural (Fairclough, 2015). This is what Mikhail Bakhtin referred to as "utterances," conceptions or assumptions that are embedded, naturalized and accordingly rendered invisible within cultural visual texts (as cited in Noble, 2013, para. 27). CDA illuminates this process, the ways in which we are often "manipulated unwittingly by the text" (O’Halloran, 2003, p. 2). CDA further investigates how text and visual media circulated in the social and political work to establish, maintain and reproduce social relations of power, dominance and inequality (Fairclough, 2015).

This study will apply Fairclough's (2015) three dimensions or stages of CDA. This first dimension is "description," which entails the formal properties or facets of text (p.58). This includes but is not limited to a text's word choice, metaphors, grammar, sentence structure and punctuation (Fairclough, 2015). The linguistic features of texts work to build ideological, and often strategic, narratives around subjects, objects, topics and ideas (Fairclough, 2015). 
Fairclough's (2015) second CDA dimension is interpretation, which regards discourse as a social practice, embedded with ideologies, and consistently subjected to "the social conditions of interpretation" (p. 57). This dimension further regards language as a socially mediated tool of power in signifying and circulating certain cultural ideologies and values to the recipient (Fairclough, 2015). This is a pivotal stage for corporate responses and communications, as interpretation of corporate language and communications directly influences the future success and public conceptions of the corporation. Fairclough (2015) asserts individuals call on their own socially generated "members resources (MR)," or personal understanding of the world, to interpret discourse (p. 57). The ways in which Amazon's communications are ideologically constructed then clearly illuminates which social groupings they are attempting to appeal to and those they are not.

Fairclough's (2015) last stage or dimension of CDA is explanation, which speaks to discourse's role in the formation, maintenance and reproduction of social structures and relations of power. For this study, this stage is significant as it illuminates how communications from those in power work to establish, produce and reproduce existing ideologies and global realities. This stage further maintains the management of discourse as a hidden method of asserting power, in which the information selected to appear in public discourse directly impacts how we understand the events, subjects and technologies around us. Similar to Black feminist approach to technology as described by Noble (2018), this dimension situates discourse and the knowledge it produces as intrinsically tied to social and political racial hierarchies and relations.

Critical discourse analysis will allow this study to uncover how Amazon's FRT communications work to reproduce racist hegemonic relations of power and silence claims of anti-black racism within FRT and Rekognition. For the purposes of this study, Amazon's general 
description of Rekognition and their specific response to allegations of racial bias in Rekognition brought forward by American Civil Liberties Union Foundation (ACLU) in their study "Amazon Teams Up with Government to Deploy Dangerous New Facial Recognition Technology” (Cagle \& Ozer, 2018) will be critically analyzed.

\section{Data Collection:}

The first piece, titled "The Facts on Facial Recognition with Artificial Intelligence" describes the functionalities, use, application and safety of Amazon's FRT, Rekognition. This piece was found through a basic Google search on Rekognition. The second piece is a response blog titled, "Thoughts on Recent Research Paper and Associated Article on Amazon Rekognition" (2019), is written by Matt Wood, Amazon's General Manager of Artificial Intelligence, in direct response to the release of ACLU's study that proved racial bias within Rekognition (Cagle \& Ozer, 2018). This piece is the most cited and discussed piece of Amazon FRT and Rekognition communications in mainstream American media as ACLU's study was further published in combination with MIT (Massachusetts Institute of Technology) in the New York Times. These two pieces were selected to highlight that both in their general and specific communications, Amazon's evasive, strategic and ideologically driven communications practices work to maintain and reproduce existing relations of power. 


\section{Section 5: Findings}

This section will discuss findings of a critical discourse analysis (CDA) of Amazon's description of their facial recognition technology, Rekognition, "The Facts on Facial Recognition with Artificial Intelligence" (2020), and their response to allegations of racial bias in Rekognition, “Thoughts on Recent Research Paper and Associated Article on Amazon Rekognition” (2019). I will first show how Amazon's use of metaphors, expressive values and relational value diminishes the detrimental implications of racial bias in FRT and law enforcement. Utilizing Hall's theory of communication as cultural exchange (1997) and Noble's (2018) discussion of technology as neutral, I will then illustrate how Amazon's communications effectively appeal to and reproduce dominant ideologies of Blackness, technology and law enforcement agencies. Applying Fairclough's (2015) three-dimensional approach to CDA, I illuminate here the hidden ideologies of what I will refer to as "discourses of racial neutrality”. Finally, I will highlight the ways in which Amazon's management of their communications is an assertion of hidden power, that reflects and reinstates dominant social and cultural values and existing relations of power (Hall, 1997; Noble, 2018).

\section{Metaphors to Obscure Claims of Racial Bias in Rekognition}

The use of metaphors is an important site of critical discourse analysis as alternative metaphors have differing ideological attachments (Fairclough, 2015). Metaphors can be understood as "representing one aspect of experience in terms of another" (Fairclough, 2015, p.137). In "The Facts on Facial Recognition with Artificial Intelligence," the descriptive piece under the subtitle "How should I apply facial recognition responsibility?" lies this metaphor:

(General, 1) "Machine learning is a very valuable tool to help law enforcement agencies, and while being concerned it's applied correctly, we should not throw away the oven 
because the temperature could be set wrong and burn the pizza" (Amazon Web Services, 2020, para.7).

Metaphors take dominant interests expressed in dominant discourse as the interests of a whole society, rather than a reflection of the interests of those with power (Fairclough, 2015). As discussed in Section 2, FRTs have the highest capacity for abuse and misuse for Black Americans and in Black communities who experience manufactured poverty and are often overpoliced and surveilled. Most Black people who experience a false stop, arrest or investigation due to FRT error or misuse would not describe the experience as the equivalent to "burning a pizza" (passage 1). Importantly, there is no mention of racial bias, racism, anti-Black racism or the specific concerns of bias made in the ACLU paper in this section or piece (more on that in dimension three). However, this lack of context and specifics around this metaphor implies the implications of FRT's racial bias are just as simple or consequential as burning a pizza, as there is no other point of reference. This metaphor further supports the Western neoliberal project, as it commodifies the personhood, identity and experience of those who experience racial bias in the development and application of FRT in law enforcement (Gates, 2011).

In addition, alternative metaphors determine different required outcomes or remedies (Fairclough, 2015). For example, an occurrence described as "like a cancer" should be eliminated with quick action before it spreads; in comparison to "like a storm" which implies a temporal nature that will eventually end on its own. In Amazon's response blog "Thoughts on Recent Research Paper and Associated Article on Amazon Rekognition,” they state:

(Specific, 2) "Amazon Rekognition is commonly known as facial recognition. It is a distinct and different feature from facial analysis and attempts to match faces that appear similar. This is the same approach used to unlock some phones, or authenticate somebody entering a building, or by law enforcement to narrow the field when attempting to identify a person of interest. In the latter, it's the modern equivalent of detectives in old movies flicking through books of photos, but much faster" (Wood, 2019, para. 3). 
ACLU's study, which passage 2 was written in response to, describes the same system, Rekognition, as:

(ACLU) "With Rekognition, a government can now build a system to automate the identification and tracking of anyone. If police body cameras, for example, were outfitted with facial recognition, devices intended for officer transparency and accountability would further transform into surveillance machines aimed at the public... As with other surveillance technologies, these systems are certain to be disproportionately aimed at minority communities" (Cagle \& Ozer, 2018, paras. 6-8).

The required reaction or remedy to "detectives in old movies flicking through books of photos, but much faster," (excerpt 2) in comparison to "a system to automate the identification and tracking of anyone" (Cagle \& Ozer, 2018, para. 6) are dramatically and, strategically on the part of Amazon, different. To support their claims the ACLU cites Amazon's internal communications and Rekognition "promotional materials (that) previously recommended that law enforcement use Rekognition to identify people in police body camera footage... The company (Amazon) removed mention of police body cameras from its site after the ACLU raised concerns in discussions with Amazon" (Cagle \& Ozer, 2018, para. 10). Figure 6 shows an image that was once publicly available on Amazon's website; it was intended to act as a guide for police officers who are using Rekognition. The image was subsequently deleted, after the ACLU study was published. The mention of "officer body cams," that "capture video footage of a person of interest or suspect during a crime scene," without their consent and Rekognition's capacities to "stream (images and data about citizens without consent) across thousands of police officers," without mention of thresholds or FRT practical ethics all work to tell a different story of Rekognition than described in the aforementioned metaphors (as cited in Cagle \& Ozer, 2018 para. 3). Amazon then does not actually believe Rekognition is "the modern equivalent of detectives in old movies flicking through books of photos, but much faster" (excerpt 2). This 
metaphor diminishes historic anti-Black practices of American law enforcement officers, including "detectives" (AWS, 2019, para. 3), such as brutal enforcement of Jim Crow laws, stop and frisk and COINTELPRO, an FBI operation, whose purpose was to surveil, discredit and neutralize Black activist groups, organizations like the Black Panthers and their supporters (Hoerl \& Ortiz, 2015). By regarding law enforcement officers and their practices as innately harmless this metaphor aims to discount the real concerns of racial bias and surveillance without consent in Rekognition brought up by the ACLU, other whistleblowers of racial bias in FRT, and those who experience racial bias and anti-Black racism in FRTs use by law enforcement.

\section{Figure 6:}

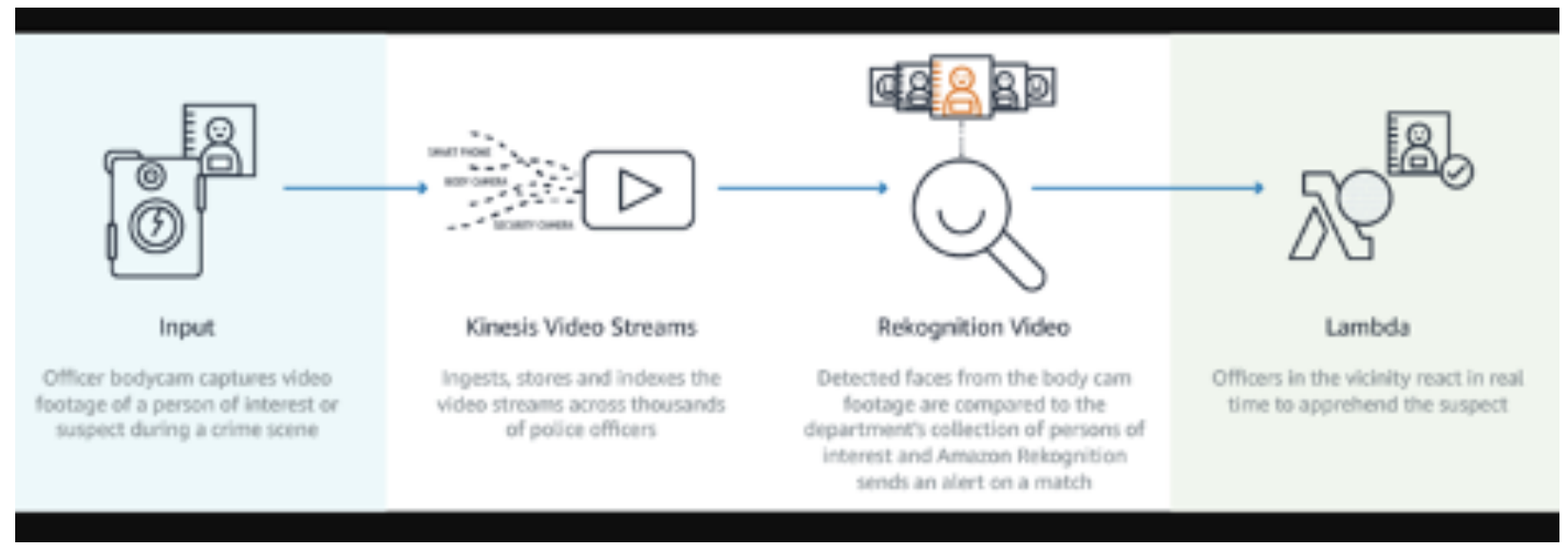

This figure illustrates promotional material for police officers who use Rekognition and it was deleted after claims of racial bias in Rekognition were made public by the ACLU (2018). Retrieved from Cagle, M., \& Ozer, N. (2018). Amazon Teams Up with Government to Deploy Dangerous New Facial Recognition Technology. ACLU.

Racial bias in the production and application of Rekognition is further obscured within Amazon's response to ACLU's study as the professional or academic associations, distinctions or accolades of the researchers at ACLU are never mentioned. Throughout Amazon's response (2019) to ACLU's study, the study is referred to as nameless and without authority. It is simply 
referred to as a "research paper" (para. 1), "latest report and its erroneous claims" (para. 7), "associated article" (para. 1). This is also seen in the title "Thoughts on Recent Research Paper and Associated Article on Amazon Rekognition." By failing to acknowledge that the study was collectively produced with input from ACLU, MIT (Massachusetts Institute of Technology), employers and officials of Washington County, FRT experts and was published in The New York Times, Amazon further erases and belittles claims of racial bias within the production and application Rekognition.

\section{A Glitch in the System: Discourses of Racial Neutrality}

\section{Relational and Expressive Value}

Throughout their general and specific Rekognition communications, Amazon refers to possibly for error within FRT as uncharacteristically possible or minuscule in its consequences, rather than an encoded aspect of all forms of FRT that targets and has life-altering implications for low-income and over-policed Black communities. Amazon uses relational value to draw more focus on Rekognition's possibility for improvement over its racial bias. Relational value speaks to a text's choice of words illuminating a perceived trust or solidarity with the readership based on apparent shared values or ideologies (Fairclough, 2015). This is evident in Amazon's general Rekognition piece," The Facts on Facial Recognition with Artificial Intelligence” (2020) under the subtitle "Is facial recognition safe?" where they state:

(General, 3) "First, some believe that people can match faces to photos better than machines. However, the National Institute for Standards and Technology (NIST) recently shared a study of facial recognition technologies that are at least two years behind the models used in Amazon Rekognition and concluded that even older technologies could outperform human facial recognition capabilities" (Amazon Web Services, 2020, para. 19). 
The possibility for Rekognition to "outperform human facial recognition capabilities" is regarded as more favourable than conceptions "that people can match faces better than machines" (excerpt 3). Additionally, naming an outlet of perceived authority, NIST, also gives the first claim more authority over the nameless "some" author in the first.

This is further seen under the same subtitle of the same piece, "The Facts on Facial Recognition with Artificial Intelligence:"

(General, 4) "As in all probabilistic systems, the mere existence of false positives doesn't mean facial recognition is flawed. Rather, it emphasizes the need to follow best practices, such as setting a reasonable similarity threshold that correlates with the given use case. Also, one of the advantages of this technology is that it continuously learns and improves, so false positives can be reduced over time" (Amazon Web Services, 2020, para. 20, my emphasis).

The italics texts highlight expressive values within the wording, which is the use of differing words that are embodied with differing sets of values (Fairclough, 2015). Expressive values can be further reduced to various discourse types or "ideologically constative classification schemes" with varying embedded values (Fairclough, 2015, p. 136). The many positive expressions in excerpt 4 illuminate and draw more focus to the possibilities and "advantages" of Rekognition for the reader and the use of negative expressions diminish Rekognition's shortcomings. This use of expressive values also works to obscure and silence claims of Rekognition's proven disproportionate error rate on Black populations and those who are dark-skinned in comparison to their white men counterparts (as discussed in Section Two). What is described in excerpts 3 and 4 are also the only concerns of Rekognition discussed in the "The Facts on Facial Recognition with Artificial Intelligence" piece, in the "Is facial recognition safe? section which starts with:

(General, 5)" Yes. Let's examine some common misconceptions about facial recognition and how it works" (Amazon Web Services, 2020, para. 12) 
And ends with

(General, 6) "Today, many successful customers, such as Thorn, VidMob, Marinus Analytics, and POPSUGAR are using facial recognition in simple ways that have a powerful impact" (Amazon Web Services, 2020, para. 16).

Claims of racial bias in Rekognition are again obscured here as it is not specifically mentioned as a "common misconception" (excerpt 5) even though it is the most documented and discussed FRT concern within FRT studies. Drawing the reader's focus to Rekognition's possibilities, perceived advantages and "powerful impact" (excerpt 6) rather than to Rekognition's racial bias further silences and mitigates claims of racial bias in Rekognition and those who experience it as equivalent to burnt pizza. Amazon therefore positions, through the use of expressive and relational values, possibilities for "best practices, improve(ment), learn(ing) and reasonable thresholds" (excerpt 4) in Rekognition as all more possible and worthy of the reader's attention than the devastating racially biased and anti-Black consequences discussed in Section Two, which the technology has proven to produce (as documented in Buolamwini et al., 2020; Buolamwini \& Gebrum, 2018; Garvie, Bedoya \& Frankle, 2016; Introna \& Nissenbaum, 2010; Learned-Miller et al., 2020).

\section{Appeal to and Support of Dominant Ideologies around Law Enforcement and Black People}

Amazon's Rekognition communications, both general and specific, appeal to those who subscribe to dominant ideologies around law enforcement agencies and Black people alike. This can be seen specifically in the following excerpt from "Thoughts on Recent Research Paper and Associated Article on Amazon Rekognition:"

(Specific, 7) It is not possible to interpret the quality of either facial analysis or facial recognition without being transparent and thoughtful about the confidence threshold used to interpret the results.... When using facial recognition to identify persons of interest in an investigation, law enforcement should use our recommended $99 \%$ confidence threshold (as documented), and only use those predictions as one element of the investigation (not the sole determinant)" (Wood, 2019, paras. 5-6). 
Additionally, in the general Rekognition description piece "The Facts on Facial Recognition with Artificial Intelligence:"

(General, 8) "Facial recognition should never be used in a way that violates an individual's rights, including the right to privacy, or makes autonomous decisions for scenarios that require analysis by a human. Regarding public safety and law enforcement, we think that governments are free to work with law enforcement agencies to develop acceptable use policies for facial recognition technologies that both protects the rights of citizens and enables law enforcement to protect the public's safety" (Amazon Web Services, 2020, para. 6).

(General, 9) "If you suspect that Amazon Rekognition is being used in manner that is abusive or illegal, or infringes on your rights or the rights of other people, please report this use and AWS will investigate the issue" (Amazon Web Services, 2020, para. 17).

In excerpts 4-9 Amazon supports and reproduces dominant American ideologies around law enforcement agencies. Law enforcement agencies have, historically and dominantly, been produced and reproduced across mediums and media of authority, such as TV, movies, news media and even history books, as "good" and even in some cases as saviours to Black populations (Hall, 1997). Hall's conception of representations as the site of meaning production reminds us that the meaning of Blackness is not fixed; rather, it is produced through representations. This becomes clear when we consider how dominant groups' ways of thinking, feeling and speaking about Black people can move across global location and culture (Hall, 1997). For example, populations across the world who have never met a Black person, yet still have an idea of what Black people are "like". This is pivotal for this study as dominant conceptions produce ideology which in turn shape dominant behaviours and accordingly the realities of given populations (Fairclough, 2015). Since the enslavement of African Americans, Black people have, dominantly and consistently in the US, been represented as naturally felonious and threatening throughout dominant narratives, texts and images. Throughout American history, in various public sites of discourse formation with authority we see Black 
people are dominantly represented as violent criminals and thugs whose advancement relies on their White counterparts, especially those in power. Whether that be law enforcement agencies, slave owners or child protective agencies. Similarly, in diversity and inclusion discourse, the idea of mentorship of Black professionals from their White counterparts is conceptualized as necessary to address what Shana Almeida (2019) refers to as, their own innate racial lack, rather than structural racial exclusion. Law enforcement agencies and the companies that produce their technology, such as Amazon, are then empowered as necessary well-intentioned beneficial saviours to Black communities, as Black people's racial lack would cause them to return to their natural state of barbaric and uncivilized violence without some kind of state intervention (Almeida, 2019). Amazon's responses in relation to racial bias in Rekognition thus joins a long history of discursive formations that work to maintain existing institutional anti-Black realities and relations of power.

The conception of Whiteness and those in power (law enforcement agencies) as civilized and innately good can only exist by consistently producing Blackness and Black people against it, as its opposite (savage, criminal and uncivilized). Said (1979) discusses these ideas in his conceptualization of Orientalist discourse, specifically how "European culture gained in strength and identity by setting itself off against the Orient as a sort of surrogate and even underground self' (p. 3). Dominant Orientalist narratives across Western mediums then falsely produce Eastern countries as "Other," as politically, socially and culturally inferior (Said, 1979). This allows the West and Western culture's authority and superiority to be maintained (Said, 1979). Similarly, the dominant conception of Black people as naturally violent throughout American dominant discourse and ideologies, allows existing anti-Black relations of power to 
persist and for the dominant conception law enforcement agencies who disproportionately incarcerate Black people as acting for the greater good.

The idea that law enforcement agencies could or would then knowingly use technology that is proven to be racially biased, and use anything less than a 99\% threshold, without good reason, is then seemingly unbelievable. Black populations who experience bias at the hands of police officers who misuse FRT, accordingly face much higher burden of proof in asserting their own lived experiences, in comparison to a White counterpart claiming for example, that a Black person committed a crime against them. One is deemed as natural or normal, whereas the other is not (Hall, 1997). The disproportionate incarceration of Black people is then deemed appropriate (or as worrisome as burning pizza). Higher crime and arrests rates are also then conceived as the fault of Black people, rather than attributed to the anti-Black racism of officers or proven racial bias in the FRTs they use. Best practices, "transparent and thoughtful" use of 99\% thresholds (excerpt 7), directive assertions made by Amazon for Rekognition to "never be used in a way that violates an individual's rights" (excerpt 9) and calls for state partnerships for legislations discussed in excerpt 8 could potentially address racial bias in Rekognition if they were applied in a system and greater society that is not dominantly and historically racially biased and antiBlack. As discussed earlier, Black populations experience disproportionate arrest, stop and investigation rates at the hands of police officers in the U.S. and as such they dominate existing law enforcement databases that Rekognition relies on. This is despite evidence they are no more likely to commit crimes or engage in criminal activity than any other demographic (Coles \& Powell, 2019). However, this is never mentioned by Amazon, even beyond the examined texts, nor is it reflected in the dominant ideologies about law enforcement Amazon supports in these 
excerpts. Instead, in Amazon's specific response to claims of racial bias (in "Thoughts on Recent Research Paper and Associated Article on Amazon Rekognition”) are:

(Specific, 10) "We know that facial recognition technology, when used irresponsibly, has risks. This is true of a lot of technologies, computers included. And, people are concerned about this. We are, too. It's why we suspend people's use of our services if we find they're using them irresponsibly or to infringe on people's civil rights. It's also why we clearly recommend in our documentation that facial recognition results should only be used in law enforcement when the results have confidence levels of at least $99 \%$, and even then, only as one artifact of many in a human-driven decision.

But, we remain optimistic about the good this technology will provide in society, and are already seeing meaningful proof points with facial recognition helping thwart child trafficking, reuniting missing kids with parents, providing better payment authentication, or diminishing credit card fraud. And, to date (over two years after releasing the service), we have had no reported law enforcement misuses of Amazon Rekognition" (Wood, 2019, para. 15).

Throughout excerpt 10, Amazon positions themselves as "concerned" with injustice and willing to suspend those who participate in it. However, as noted in Section 2, police officers in Washington Country were using Rekognition on average twenty times a day without the knowledge and consent of the public (Washington Post, 2020). By claiming "we have had no reported law enforcement misuses of Amazon Rekognition” (excerpt 10) Amazon further supports the conception of Black people, who experience racial bias in FRT the most, as innately violent, troublesome or criminal (Garvie, Bedoya \& Frankle, 2016). This passage contributes to the large-scale silencing of claims of anti-Black racism and violence as it supports the dominant ideology that police and law enforcement agencies act in the best interest of all populations and would report it if they or their colleagues did not.

Fairclough (2015) additionally regards whose perspectives or observations are included in media intended for the masses as an effect of power. Importantly, throughout all of Amazon's examined texts, there are only mentions of positive experiences and results with Rekognition. In 
this, those who experience the effects of anti-Black racism that is inherent to the very reasons behind the use and development of Rekognition are further silenced.

\section{Amazon as an FRT Authority}

What Amazon fails to discuss or acknowledge is FRTs, like all AI-powered technology, work to disseminate and reproduce existing anti-Black structures within law enforcement agencies, which cannot be changed with "best practices" (Noble, 2018). Additionally, even if anti-Black structures and practices in law enforcement were somehow mitigated, Rekognition's use could still be racially bias or flawed as FRT's application in law enforcement is too varied to be accounted for by national legislation, operational thresholds or best practices (Learned-Miller et al., 2020). This is well established in FRT scholarly discourse, but never addressed by Amazon. Moreover, the possibility for Rekognition to gradually have "false positives can be reduced over time" could be seemingly possible. However, as discussed in Section Two, FRTs base algorithms become further racially biased based on their interactions with training data that regard racial bias as correct. Amazon is perceived as an FRT authority, an objective evaluative body that should receive any and all Rekognition issues (as stated by Amazon in excerpt 9). When members of the public then seek knowledge from digital platforms or receive information or communications from technology producers it is perceived as an authority on truth (Noble, 2018). Similar to mechanical objectivity, perceived technological objectivity allows producers of technology with power like Amazon to decide what truly is, or is objective, based on their own interests or coded gaze (Buolamwini, 2018).

Amazon utilizes this perceived authority to reinstate the disproportionate arrests rates and over-policing of Black communities by law enforcement agencies who use FRTs as natural by asserting Rekognition has the capacity to improve with greater use. Communications from those 
in power can and do shape existing realities (Fairclough, 2015). Hall (1997) therefore calls for a strong analysis of larger units, the "narratives, statements, groups of images... which operate across areas of texts, areas of knowledge about a subject which have acquired widespread authority" (p. 39). Public, dominant or mainstream discourse enables how members of a shared culture behave and regulate behaviour, as all social practices and configurations carry societal meaning (Hall, 1997). Dominant discourse establishes, maintains and naturalizes "certain ideological assumptions as commonsensical" or the "truth of the matter," (Fairclough, 2015, p.113; Hall, 1997). "Naturalization is the royal road to common sense" as when an assumption or ideology is regarded as natural or with a fixed meaning that is an "effect of power" (Fairclough, 2015, p. 113).

In order to maintain existing relations of power, dominant discourses must then be repetitive in nature and embody the familiar and historical ideologies of those in power (Hall, 1997). Knowledge of a certain group is not thus developed in isolation, it is based on cultural "general politics of truth" (Hall, 1997, p. 49). To truly understand a given topic or subject we must then look at all discourses, including those "that challenge functionalist and systematic thought that are masked or disqualified by those in power" (Foucault, 1980, p. 81). For example, the Black Panther Party, a Black political activist group formed in 1966, founding values included employment, land, housing and justice for all (Russonello, 2016). Yet, more than fifty years after their conception, they are still predominantly remembered as one of "the greatest threats to (American) internal security" or "beret wearing, angry revolutionaries with big Afros and guns," as this narrative dominated discourse of the time (Russonello, 2016, paras. 5-11). The Black Panther's multi-generational, multi-racial and community-based activism challenged existing and hegemonic relations of power and was consequently removed from mainstream 
discourse. Dominant discourse has long worked to systematically oppress Black and marginalized communities' realities through privileging certain ideologies and narratives and minimizing others. Therefore, Amazon's dissemination of discourses of racial neutrality has direct implications on the realities of Black populations as it erases, and silences claims of antiBlack racism at the hands of law enforcement agencies. As the disproportionate arrest rates of Black Americans and the detrimental consequences of FRT use and surveillance in Black communities are both deemed natural, and arguably in the best interest of the maintenance of normal American life, claims of racial bias in Rekognition are thereby discredited as a glitch in the system, rather than ingrained within the system's very nature.

\section{Discourses of Racial Neutrality}

Throughout the examined texts and in the aforementioned excerpts, Amazon enables what I refer to as discourses of racial neutrality, in which a corporation positions itself as outside of, beyond and/or immune to racially biased and/or anti-Black relations of power. This builds off of what Noble (2018) refers to as "ideologies of colorblindness," which insincerely signify a humane and nonracist worldview where "celebrations of multiculturalism and diversity obscure structural and social oppression... which are shaping technological practices" (p. 167).

Discourses of racial neutrality further allows for instances of racism to be deemed as based on an individual's personal opinions or conceptions rather than a product of systemic racism throughout our society or, in this case, a glitch in the system. This conception works to further silence and erase claims of racial bias and anti-Black racism within FRTs use in law enforcement. The public conception of the fairness and objectivity of technology in a colourblind society allows perpetrators of algorithmic oppression and racial bias with power, like Amazon, to avoid being held accountable-even in the face of evidence of proven racial bias and 
anti-black racism in the production and application of their AI-powered technologies (Noble, 2018). Members of a given society are more susceptible to social control under discourses of "control by consent," or simulated egalitarianism and the absence of outward markers of authority, than traditional methods of control by coercion (Fairclough, 2015, p. 67). This is because members of a society feel a part of rather than against "apparatuses of control" through discourses of an apparent shared democracy (Fairclough, 2015, p. 67). This is evident in America and Canada alike; society is produced by those in power as equal for all. Amazon employs discourses of racial neutrality in their claim that there has been "no reported law enforcement misuses of Amazon('s) Rekognition" (excerpt 9) in combination with their assertion that "facial recognition technology, when used irresponsibly, has risks...but we (Amazon) remain optimistic about the good this technology will provide" citing various "meaningful proof points" (excerpt 9). This word choice further positions the possibility for error, racial bias and anti-Black racism within Rekognition as an unlikely or uncharacteristic glitch rather than an entrenched aspect of the technology and its application within law enforcement.

\section{Amazon positioned as Ally to the Racial Other}

In both texts, Amazon also positions themselves as against civil injustices and outside of the scope of relations of racism. This is seen in their response piece "Thoughts on Recent Research Paper and Associated Article on Amazon Rekognition":

(Specific, 11) "The research paper implies that Amazon Rekognition is not improving, and that AWS is not interested in discussing issues around facial recognition...This is false. We are now on our fourth significant version update of Amazon Rekognition. We are acutely aware of the concerns around facial recognition, and remain highly motivated and committed to continuous improvement, just as we are with all of our services. We make funding available for research projects and staff through the AWS Machine Learning Research Grants and have made significant investments to continuously improve Amazon Rekognition" (Wood, 2019, paras. 13-14, my emphasis). 
Engaging with discourses of racial neutrality is advantageous for corporations like Amazon because it allows them to appeal to those in power and those who support existing relations of power. Excerpt 11 illustrates Amazon's recirculation of dominant colour-blind ideologies. Subscribing to and supporting such ideologies further allows Amazon to avoid responsibility for racial bias while maintaining racist and profitable practices. As within such ideologies, only the worst possible kinds of race relations, events or instances that no one can deny or contest are racially motivated and can be deemed as racist or fueled by racism. An important aspect of discourses of racial neutrality is positioning the corporation as against civil injustices and racial bias. Responsibility to fix issues of race or racism is then firmly put on those who are racialized, especially when they are seeking remedies from systematic racist practices or power relations, such as racial bias in Rekognition and its use in law enforcement (Noble, 2018). The possibility for accountability and reparations in such circumstances then becomes further challenging for racialized people as the possibility of mass empathy and understanding becomes impossible (Noble, 2018).

The italicized text in excerpt 11 highlights expressive values within word choice employed to signify an apparent commitment to improving potential, but unnamed, shortcomings or possibilities for misuse of Rekognition. Upon closer examination it is clear that there is no actual commitment to action or tangible strategies of improvement made. Phrases such as "highly motivated," “committed," "improvement," and “investments," (passage 11) are hollow words that signify an apparent commitment without a clear path of action or accountability. Moreover, the funding discussed here is to AWS, which is Amazon Web Services, the owner of Rekognition. As discussed in Section 2, it is problematic that FRT producers like Amazon are positioned as the sole authority and evaluators of their own technology as future developments 
are further embedded with their own coded gaze (Buolamwini, 2018). This is further seen in the

"Thoughts on Recent Research Paper and Associated Article on Amazon Rekognition"

response piece:

(Specific, 12) "Those improvements are made available to customers in all geographic regions, as soon as our improvements are validated - and just like all AWS services - we will continue to update and improve Amazon Rekognition. So far, our direct offers to discuss, update, and collaborate on these results have not been acknowledged or accepted by the researchers in this case" (Wood, 2019, para. 14).

Those who experience and assert racial bias within Rekognition are referred to here, overtly and covertly, as not working in the best interest of society, devise or unwilling to "discuss, update and collaborate on these results" (excerpt 12). This works to further produce those who experience and assert racial bias within FRT and Rekognition application in law enforcement as the racial Other (Almeida, 2019) and Amazon as committed to social good.

\section{Erasure of anti-Black Realities}

Amazon further enables discourses of racial neutrality as within the examined text, there is no mention of existing anti-Black relations of power. Additionally, Amazon fails to acknowledge historic anti-Black practices of law enforcement agencies, over-representation of Black people in the databases Amazon's Rekognition functionality relies on, or FRT's proven disproportionate high error rate on Black populations that are all widely established in FRT and AI-technology literature (as cited in Section Two). This erasure can be seen in this expert of Amazon's general piece, "The Facts on Facial Recognition with Artificial Intelligence:"

(General, 13) "Facial recognition is a system built to identify a person from an image or video. This technology has been around for decades, but its usage has become more noticeable, and accessible, in the past few years as it now powers innovative solutions, such as personal photo applications and secondary authentication for mobile devices" (Amazon Web Services, 2020, para. 2). 
Facial recognition technology has been around for decades, and it has been already utilized to target Black communities for decades, something which this passage fails to mention.

This is further supported in this excerpt from the same piece

(General, 14) "Facial analysis capabilities, such as those available in Amazon Rekognition, allow users to understand where faces exist in an image or video, as well as what attributes those faces have. For example, Amazon Rekognition can analyze attributes such as eyes open or closed, mood, hair color, as well as the visual geometry of a face. These detected attributes become increasingly useful for customers that need to organize or search through millions of images in seconds using metadata tags (e.g., happy, glasses, age range) or to identify a person (i.e., facial recognition using either a source image or a unique identifier)" (Amazon Web Services, 2020, para. 3).

Passage 14 discusses facial attribute estimation, a process within FRT discussed in Section Two, that has been proven to have highest error rates and possibilities for misunderstanding of perceived emotions for Black Americans (Buolamwini et al., 2020). However, that is not mentioned here. As Fairclough (2015) asserts, to have the power to constrain content within discourse to favour certain perspectives and "wordings of events" while removing others is an assertion of hidden power (p. 80). Excerpt 14 and 15 further positions those who assert and experience racial bias in Rekognition's application in law enforcement as against existing social relations or looking for problems that are seemingly not there. This conception plays a role in the large-scale silencing of anti-Black racism as it takes away culturally perceived authority, validity and trust of Black voices. Only by publicly highlighting intersectional relations of power will we be able to transform the "consciousness of artificial intelligence since it is, in part, a product of our own collective creation" (Noble, 2018, p. 29). By not acknowledging intersectional and dominant anti-Black relations of power in their responses to allegations of racial bias in Rekognition, Amazon works to uphold this very consciousness. Amazon utilizes their authority to decrease the validity of the interests of those who experience and assert racial bias in Rekognition and FRTs by never actually directly responding to or 
naming these concerns. As Fairclough (2015) discusses, discourse works to continuously legitimize and re-legitimize existing realities and relations of power. As discussed, technology and it's producers like Amazon are dominantly understood and portrayed within dominant discourse as neutral or racially neutral. This conception paired with the use of discourses of racial neutrality that erase racist and anti-Black relations of power allows Amazon, as a perceived FRT authority, to produce the use of Rekognition in the targeting, further criminalizing and incarcerating Black communities as not only a glitch in the system that is the equivalent to burning pizza but also as a natural occurrence for the better of society that has nothing to do with race. 


\section{Section 6: Conclusion}

This study employed a critical discourse analysis of Amazon's general description of Rekognition and specific response to ACLU's allegations of racial bias in Rekognition to argue that Amazon works to obscure racial bias in both development and application of FRTs in law enforcement. By appealing to and supporting dominant ideologies and narratives that persist in the US about law enforcement as innately good and Black people as naturally felonious (Hall, 1997), Amazon further obscures the experiences of Black people who experience bias and subsequent criminalization within FRTs use in law enforcement. Amazon also enables what I have referred to as discourses of racial neutrality which, combined with the conception of technology as neutral, allows Amazon to deem any racially biased FRT outcomes as a "glitch in the system." In doing so, I argue that Amazon contributes to the large-scale project of reinstating who holds power in America, and who does not by never directly speaking to racial bias in FRT or historic anti-Black racism in the law enforcement practices and systems that Rekognition relies on. As Fairclough (2015) argues, to be enabled to determine what stories and perspectives are reflected in text or media produced for the masses is an assertion and reinstation of power. Amazon's FRT communications, both general and specific, utilize Amazon's perceived authority and power to silence claims of racial bias in FRT and other AI-powered technologies and naturalize anti-Black realities and relations in law enforcement and North America at large.

Dominant discourse has the power to create, develop and change existing realities (Fairclough, 2015). Dominant representations and ideologies have additionally long had lifealtering consequences for those who are already made vulnerable by existing relations of power (Fairclough, 2015). Systematic or encoded racial bias, the erasure of claims made by already marginalized communities and the use of technology to amplify existing racist relations of power 
are not new facets or events of our societies. Technology is too often regarded as a changing, otherworldly and, as discussed, neutral force only to accordingly be investigated by experts of math and science. Algorithmic oppression (Noble, 2018), ideologies of colorblindness (Noble, 2018), the coded gaze (Buolamwini, 2018) and what have I have referred discourses of racial neutrality are crucial developments in the workings of Hall's conception of the power of representations in discourse in shaping our knowledge, behaviours and realities. This study therefore aims to add to Noble's (2018) call for a critical race theory and Black feminist approach to technology that grounds the investigation of technological development and application in existing racial hierarchies and power relations. Accordingly, as Rekognition, FRTs and AIpowered technology develop in both use and capacity in Canada, America and the world at large, it is imperative that we further investigate not only how these technologies work on given populations but also how their producers contribute and reinstate racist and anti-Black relations of power through their communications. 


\section{Section 7: Epilogue}

When I started this study, the topic of racial bias and anti-Black racism in FRTs and AIpowered technology was on the rise, but I would have never expected for the topic to take off as much as it did. It is promising and exciting that so many discussions of the direct links between technological development and relations of power are entering mainstream forms of discourse. In this Epilogue, I would like to acknowledge some of the more recent develops around the topics of FRT and discuss the ways in which many of the concerns outlined in this paper still stand. It is important to not be swayed by further discourses of racial neutrality or apparent allyship, especially in this high-stakes political moment.

In June 2020, IBM released a public letter to US Congress announcing that the company will no longer develop or research FRT and further positioning themselves against the use of FRT in "mass surveillance, racial profiling, violations of basic human rights and freedoms" (para. 9). This did act as a needed catalyst, forcing other technology producers to navigate a public sphere where FRT and AI-powered technologies innate bias has been acknowledged by a fellow technology producer with power. The following week, Amazon announced that they were putting a pause on all Rekognition research and development for police use only, for one year. However, Amazon did not acknowledge any historic or current racial bias or anti-Black racism within Rekognition. Their public announcement rather claimed the "moratorium" was to "give Congress enough time to put in place appropriate rules" (Amazon, 2020, para. 1).

Clearview AI, the biggest supplier of FRTs to police departments who famously boasted about scrapping mass amounts of photos from social media sites, then announced they would stop supplying FRT in Canada due to a privacy probe (Daigle, 2020). 
This series of news is a necessary first step in a positive direction and speaks volumes to the advocacy of organizations like ACLU and the Algorithmic Justice League. But I wish to emphasize, there is still much work to be done. Big Tech producers' role in silencing claims of racial bias and perpetuating anti-Black racism in their technology and beyond is far from over. Neither Clearview, Amazon nor IBM acknowledged their own role in perpetuation of racial bias, racism, surveillance and police violence. Too many Black and racialized people across Canada and America have lived through false arrests, investigations, harassment and violence through the application of FRT and AI-powered technologies. Until the systems in which FRTs production thrived are changed, something similar is on its way.

We must also not forget that IBM still supports ICE and Border Control with surveillance equipment (Elias, 2019). Clearview still operates and is scraping social media sites in America (ACLU, 2020). Amazon is still researching and developing FRT for use outside of police, and those developments will be quickly applied to law enforcement use of FRT once their moratorium is over. Additionally, while Big Tech companies attempt to publicly step away FRT development for the moment, small tech producers with lower profiles like NEC, Cognitec and iOmniscient are picking up where they left off (Horowitz, 2020). While we should celebrate the aforementioned achievements, we must not turn away from investigating and illuminating the ways in which Big Tech companies utilize gaps in policy to exploit and silence the most vulnerable members of our society. 


\section{Section 8: Works Cited}

A Collective Impact: Interim report on the inquiry into racial profiling and racial discrimination of Black persons by the Toronto Police Service. (2018). Ontario Human Rights Commission. http://www3.ohrc.on.ca/en/public-interest-inquiry-racial-profiling-and-discrimination-toronto-policeservice/collective-impact-interim-report-inquiry-racial-profiling-and-racial-discrimination-black

ACLU v. Clearview AI - Complaint. (2020, May). American Civil Liberties Union. https://www.aclu.org/legal-document/aclu-v-clearview-ai-complaint

Allen, K., \& Gillis, W. (2019, May 28). Toronto police have been using facial recognition technology for more than a year. Toronto Star. https://www.thestar.com/news/gta/2019/05/28/toronto-police-chiefreleases-report-on-use-of-facial-recognition-technology.html

Almeida, S. (2020). Language and Power (the basics). https://courses.ryerson.ca/d21/le/content/333822/viewContent/2663532/View.

Almeida, S. (2019). Mythical encounters: challenging racism in the diverse city. International Journal of Sociology and Social Policy, 39(11/12), 937-949. https://doi.org/10.1108/IJSSP-11-2018-0198

Amazon Web Services. (2020). The Facts on Facial Recognition with Artificial Intelligence. AWS. https://aws.amazon.com/rekognition/the-facts-on-facial-recognition-with-artificial-intelligence/

Barocas, S., \& Selbst, A. D. (2016). Big data's disparate impact. California Law Review, 104(671).

Brinckerhoff, R. (2018). Social network or social nightmare: How California courts can prevent Facebook's frightening foray into facial recognition technology from haunting consumer privacy rights forever. Federal Communications Law Journal, 70(1), 105-156.

Buolamwini, J. (2016, November). How I'm fighting bias in algorithms [TedTalk]. https://www.ted.com/talks/joy_buolamwini_how_i_m_fighting_bias_in_algorithms

Buolamwini, J., \& Gebru, T. (2018). Gender Shades: Intersectional Accuracy Disparities in Commercial Gender Classi cation. Proceedings of Machine Learning Research, 81, 1-15.

Buolamwini, J., Ordóñez, V., Jamie Morgenstern, \& Learned-Mille, E. (2020). Facial Recognition Technologies: A Primer. Algorithmic Justice League.

Cagle, M., \& Ozer, N. (2018). Amazon Teams Up With Government to Deploy Dangerous New Facial Recognition Technology. ACLU. https://www.aclu.org/blog/privacy-technology/surveillancetechnologies/amazon-teams-government-deploy-dangerous-new

Caliskan-Islam, A., Bryson, J. J., \& Narayanan, A. (2016). Semantics derived automatically from language corpora contain human-like biases. Science, 356(6334), 183-186 •

CBC News. (2020, February 13). Toronto police admit using secretive facial recognition technology Clearview AI. CBC. https://www.cbc.ca/news/canada/toronto/toronto-police-clearview-ai-1.5462785 
Coles, J. A., \& Powell, T. (2020). A BlackCrit analysis on Black urban youth and suspension disproportionality as anti-Black symbolic violence. Race Ethnicity and Education, 23(1), 113-133. https://doi.org/10.1080/13613324.2019.1631778

Daigle, T. (2020, July 6). Clearview AI stops offering facial recognition software in Canada amid privacy probe I CBC News. CBC News. https://www.cbc.ca/news/technology/clearview-ai-stops-facialrecognition-in-canada- 1.5639380

Daston, L., \& Galison, P. (1992). The Image of Objectivity. Representations, 40, 81-128. JSTOR. https://doi.org/10.2307/2928741

DeMarco, J. V. (2012). US Federal Trade Commission hosts public forum on facial recognition technology.

Doffman, Z. (2019, January 28). Amazon Refuses To Quit Selling "Flawed" And "Racially Biased" Facial Recognition. Forbes.

Elias, J. (2019, August 19). More than 1,000 Google employees implore leaders to stop working with U.S. border and immigration agencies. CNBC. https://www.cnbc.com/2019/08/19/google-employees-imploreleaders-to-stop-working-with-us-bcp-ice.html

Fairclough, N. (2015). Language and Power: Third Edition. Routledge.

Foucault, M. (1980). Two Lectures. In Power/Knowledge: Selected Interviews and Other Writings 19721977. Pantheon Books.

García-Hodges, A., Sottile, C., \& Ward, J. (2020, June 26). Man wrongfully arrested due to facial recognition software talks about "humiliating" experience. NBC News. https://www.nbcnews.com/business/business-news/man-wrongfully-arrested-due-facial-recognitionsoftware-talks-about-humiliating-n1232184

Garvie, C., Bedoya, A., \& Frankle, J. (2016). The Perpetual Line-Up: Unregulated Police Face Recognition in America. Georgetown Law Center on Privacy \& Technology.

Gates, K. A. (2011). Our biometric future: Facial recognition technology and the culture of surveillance (Vol. 2). NYU Press.

Gilbert, B. (2020, February 21). Amazon sells facial recognition software to police all over the US, but has no idea how many departments are using it. Business Insider. https://www.businessinsider.com/amazonrekognition-police-use-unknown-2020-2

Graham, S., \& Wood, D. (2003). Digitizing surveillance: categorization, space, inequality. Critical Social Policy, 23(2), 227-248.

Hall, S. (1997). Representation: Cultural Representations and Signifying Practices. Sage Publications.

Harrisburg University. (2020). Facial Recognition Software Predicts Criminality, Researchers Say. IBM CEO's Letter to Congress on Racial Justice Reform. (2020, June 8). IBM THINKPolicy Blog. https://www.ibm.com/blogs/policy/facial-recognition-sunset-racial-justice-reforms/

Hoerl, K., \& Ortiz, E. (2015). Organizational Secrecy and the FBI's COINTELPRO-Black Nationalist Hate Groups Program, 1967-1971. Management Communication Quarterly, 29(4), 590-615. 
Horowitz, J. (2020, July 3). Tech companies are still helping police scan your face. CNN. https://www.cnn.com/2020/07/03/tech/facial-recognition-police/index.html

Institute for Crime \& Justice Policy Research, \& University of London. (2019). United States of America: Overview. World Prison Brief. https://www.prisonstudies.org/country/united-states-america

Introna, L. D., \& Nissenbaum, H. (2010). Facial Recognition Technology: A Survey of Policy and Implementation Issues. The Centre for Catastrophe Preparedness and Response.

Joseph, G., \& Lipp, K. (2018). IBM used NYPD surveillance footage to develop technology that lets police search by skin color. The Intercept in partnership with the Investigative Fund. https://theintercept.com/2018/09/06/nypd-surveillance-camera-skin-tone-search/

Kleinman, Z. (2019, February 4). Amazon: Facial recognition bias claims are 'misleading'. BBC News.

Learned-Miller, E., Ordóñez, V., Morgenstern, J., \& Buolamwini, J. (2020). Facial Recognition Technologies in The Wild. A Call for Federal Office. Algorithmic Justice League.

Noble, S. U. (2013). Google search: Hyper-visibility as a means of rendering black women and girls invisible. InVisible Culture, 19.

Noble, S. U. (2018). Algorithms of oppression: How search engines reinforce racism. New York University Press.

O’Halloran, K. A. (2003). Critical Discourse Analysis and language cognition. Edinburgh University Press.

Rubel, A. (2007). Privacy and the USA patriot act: Rights, the value of rights, and autonomy. Law and Philosophy, 26(2), 119-159.

Said, E. (1979). Orientalism. Pantheon Books.

Samuel, S. (2019, April 27). The growing backlash against facial recognition tech. Vox. https://www.vox.com/future-perfect/2019/4/27/18518598/ai-facial-recognition-ban-apple-amazon$\underline{\text { microsoft }}$

Silva, S., \& Kenney, M. (2018). Algorithms, Platforms, and Ethnic Bias: An Integrative Essay. Phylon, 55(1 \& 2), 9-37. JSTOR. https://www.jstor.org/stable/26545017

Simpson, P., Mayr, A., \& Statham, S. (2018). Language and power: A resource book for students. Routledge.

Snow, J. (2018). Amazon's face recognition falsely matched 28 members of Congress with mugshots. American Civil Liberties Union.

Taneja, H. (2019, January 22). The Era of "Move Fast and Break Things" Is Over. Harvard Business Review. https://hbr.org/2019/01/the-era-of-move-fast-and-break-things-is-over

Washington Post. (2019, April 30). Amazon's facial-recognition technology is supercharging Washington County police. Washington Post. https://www.oregonlive.com/washingtoncounty/2019/05/amazonsfacial-recognition-technology-is-supercharging-washington-county-police.html 
We are implementing a one-year moratorium on police use of Rekognition. (2020, June 10). The Amazon Blog Day One. https://blog.aboutamazon.com/policy/we-are-implementing-a-one-year-moratorium-onpolice-use-of-rekognition

Wood, M. (2019, January 26). Thoughts on Recent Research Paper and Associated Article on Amazon Rekognition. Amazon Web Services Machine Learning Blog. https://aws.amazon.com/blogs/machinelearning/thoughts-on-recent-research-paper-and-associated-article-on-amazon-rekognition/ 